\title{
On the continuity of equilibrium and core correspondences in economies with differential information $\star$
}

\author{
Ezra Einy $^{1}$, Ori Haimanko ${ }^{1}$, Diego Moreno ${ }^{2}$, and Benyamin Shitovitz ${ }^{4}$ \\ 1 Department of Economics, Ben Gurion University of the Negev, Beer Sheva 84105, ISRAEL \\ (e-mail: $\{$ einy,orih\}@bgu.ac.il) \\ 2 Departamento de Economía, Universidad Carlos III de Madrid, 28903 Getafe, SPAIN \\ (e-mail: dmoreno@eco.uc3m.es) \\ 3 Department of Economics, University of Haifa, Haifa 31905, ISRAEL \\ (e-mail: binya@econ.haifa.ac.il)
}

Received: January 16, 2004; revised version: October 28, 2004

Summary. We study upper semi-continuity of the private and coarse core and the Walrasian expectations equilibrium correspondences for economies with differential information, with Boylan (1971) topology on agents' information fields.

Keywords and Phrases: Economies with differential information, Walrasian expectations equilibrium, Private core, Coarse core.

JEL Classification Numbers: D50, D82, C70.

\section{Introduction}

We study the behavior of several notions of competitive equilibrium and core in economies with differential information. Our aim is to check whether these solution concepts respond continuously to changes in agents' characteristics in the underlying economy. This is a basic problem in general equilibrium theory, that was studied by several authors in the context of economies with complete information. For instance, Kannai (1970) considered continuity properties of the core of a pure exchange economy, while Hildenbrand (1972) and Hildenbrand and Mertens (1972) investigated the continuity of the equilibrium-set correspondence. It turns out that both solution concepts are "upper semi-continuous": when the agents' characteristics converge, together with a selection from the solution of corresponding

* We wish to thank Carlos Herves, Nicholas Yannelis, and an anonymous referee for their helpful comments.

Correspondence to: E. Einy 
economies, then the limit of the selection belongs to the solution of the limiting economy.

In an economy with differential information every agent is characterized by his initial endowment of commodities (which is a random variable, whose value is determined by the realization of the state of nature), his state-dependent utility function, and his private information represented by a $\sigma$-field on the space of states of nature (i.e., an agent can tell whether the realized state of nature is contained in any given set from the field). In measuring "closeness" of these economies, the only non-standard part lies in evaluating the "distance" between agents' information endowments. We do so by means of Boylan (1971) metric, following Allen (1983) who was the first to apply topologies on information fields to economies with differential information. (Allen proved continuity ${ }^{1}$ of the consumer demand and the value of information with respect to the Boylan metric.)

We first consider a notion of competitive equilibrium for economies with differential information, the Walrasian expectation equilibrium (WEE), introduced in Radner $(1968,1982)^{2}$. In a WEE, all commodities are priced in every state of nature, and each agent maximizes ex-ante (expected) utility on his budget set, but his trades must be measurable with respect to his private information. We show that if the space of random commodity bundles is $L_{p}^{m}$ (where $m$ is the number of commodities, and $1 \leq p<\infty)$ and the prices lie in its dual, then WEE correspondence is upper semi-continuous (see Theorem 1).

We do not consider an alternative equilibrium concept, that of "rational expectations," in studying continuity of equilibrium correspondences, because the lack of continuity is apparent in this case. Indeed, in a rational expectations equilibrium agents maximize their interim utility - expected utility conditional on private information and the information revealed by prices. Information revealed by prices may, however, have noticeable discontinuity in the transition to the limiting economy. An extreme example of such a discontinuity is provided by a sequence of fully revealing equilibrium price systems that converges to a constant price system, which of course reveals no information. This discontinuity of revealed information translates into discontinuity of the rational expectations equilibrium correspondence: the limit of agent's commodity bundles in equilibrium allocations may well be a suboptimal choice for the agent given the information revealed by prices in the limiting economy.

Our second focus is on core correspondences. In the literature there are several notions of core for economies with differential information (for a comprehensive survey see Glycopantis and Yannelis, 2004; Forges et al., 2002). Among them, two notions stand out in having the important and useful property of being nonempty under quite general conditions on the economy. These are Wilson (1978) coarse core, where a blocking coalition considers its interim payoffs (following the revelation of private information to agents) given a common knowledge event, and Yannelis

${ }^{1}$ Cotter $(1986,1987)$ showed that continuity properties could be obtained with a weaker topology. Stinchcombe (1990) derived further results for both topologies. Van Zandt (2002) studied continuity of solutions to constrained maximization problems with respect to the Cotter (1986) topology on information fields.

2 The Walrasian expectations equilibrium is sometimes also referred to as Radner equilibrium. 
(1991) private core, where blocking is based on ex-ante payoffs, but measurability of all allocations with respect to agents' private information is required. We will confine our attention to these core correspondences. ${ }^{3}$

The relation between the private core and WEE is analogous to that of the core and Walrasian equilibrium in a complete information economy - see Einy et al. (2001) for an equivalence result for economies with differential information and a continuum of traders. Despite this relation, it turns out that the private core correspondence differs from the WEE correspondence in its behavior. We show here that the private core correspondence may not be upper semi-continuous even in simple economies (see Example 1). However, the private core correspondence is upper semi-continuous for every converging sequence of economies where agents' information fields approach their limiting information fields from above (i.e., in the sequence agents are better informed than in the limit); see Theorem 2.

The private core correspondence is also upper semi-continuous when the limiting economy is a complete information economy (see Theorem 3 ). This is in line with the earlier result of Balder and Yannelis (2002). They showed upper semicontinuity of the private core correspondence under very general conditions on the economies $^{4}$, but required the quality of information along the converging sequence to be monotonically increasing, which is not needed in our setup. At the same time, our Theorem 3 stands in contrast to Krasa and Shafer (2001), that showed discontinuity of this correspondence when the agents' common prior $^{5}$ over the set of states of nature changes, in a way that the information becomes complete in the end. Here we view the common prior as an invariable characteristic of the economy ${ }^{6}$ (together with the set of agents, commodities, and the states of nature), and not as part of the agents' characteristics. Accordingly, in all our results the information fields of the agents (and their other characteristics) are allowed to change, but the common

\footnotetext{
${ }^{3}$ Apart from the non-emptiness of these two core correspondences, there are more reasons for focusing attention only on them. As we shall see, neither one of these correspondences is upper semicontinuous in general, and we believe that with other core concepts, conceived in the similar spirit, the situation will likely be the same. Moreover, when incentive compatibility constraints are added to the notion of core (see Vohra, 1999), restrictive assumptions must be imposed on the way the information is revealed, in order for the "reports" of agents to be well defined. In our setup, these restrictions take the following form: the information fields would have to be generated by partitions of the space of states of nature (a report is then simply an element of the paritition). Accordingly, an element of the incentive compatible core, which is a mechanism defined on products of all reports, changes its domain when the information endowments (the partitions) in the economy undergo changes. This introduces an additional difficulty in studying the behavior of this core correspondence, since it is not obvious how to define convergence of incentive compatible mechanisms with changing domains.

4 Balder and Yannelis (2002) only require weak convergence of core allocations in the sequence, while we need the $L_{1}^{m}$-norm convergence (see Sect. 2). Also, they work with significantly weaker assumptions on the space of states of nature and the utility functions, and allow for state-dependent consumptions sets.

5 To interpret the model of Krasa and Shafer (2001) in our setting, one has to view this common prior as defined on the product of the set of states of nature and the signals receivable by agents, rather than the set of states of nature alone (as we do here, with information fields as a device of revealing information).
}

${ }^{6}$ Or, more precisely, of its information structure determined by the underlying economic data. 
prior stays fixed. This is precisely what allows us to obtain the positive result of Theorem 3.

Finally, we examine the coarse core correspondence, and find that it is also fragile, and may fail to be upper semi-continuous even when the private core correspondence is (see Example 2).

\section{The model}

We consider a pure exchange economy $\mathcal{E}$ with differential information. The commodity space is $R_{+}^{m}$. The set of agents is $N=\{1,2, \ldots, n\}$. The uncertainty in the economy is described by a probability space $(\Omega, \digamma, \mu)$, where $\Omega$ is the space of states of nature, which we assume to be compact and metrizable, $\digamma$ is a $\sigma$-field of subsets of $\Omega$, and $\mu$ is the common prior of the agents - a (countably additive) probability measure on $(\Omega, \digamma)$. Private information of agent $i \in N$ is given by a $\sigma$-subfield $\digamma^{i}$ of $\digamma$; that is, for every $A \in \digamma^{i}$ agent $i$ knows whether the realized state of nature is contained in $A$.

For any $1 \leq p<\infty$ we write $L_{p}^{m}$ for the Banach space of all $\digamma$-measurable functions $^{7} x: \Omega \rightarrow R^{m}$ such that $\|x\|_{p} \equiv\left(\int_{\Omega}\|x(\omega)\|^{p} d \mu(\omega)\right)^{\frac{1}{p}}<\infty$ (here $\|\cdot\|$ stands for the Euclidean norm on $R^{m}$ ). It is well known that $L_{p}^{m} \subset L_{1}^{m}$ and that convergence in the $L_{p}^{m}$-norm implies convergence in the $L_{1}^{m}$-norm.

Given $S \subseteq N$, an $S$-assignment in economy $\mathcal{E}$ is an $S$-tuple $\mathbf{x}=\left(x^{i}\right)_{i \in S}$ of non-negative functions (commodity bundles) in which $x^{i} \in L_{1}^{m}$ for every $i \in S$. An $N$-assignment is simply referred to as assignment. There is a fixed initial assignment of commodities in the economy, $\mathbf{e}=\left(e^{1}, \ldots, e^{n}\right) ; e^{i}$ is referred to as the initial endowment of agent $i$. An $S$-allocation is an $S$-assignment $\mathbf{x}=\left(x^{i}\right)_{i \in S}$ that $^{8}$ satisfies the feasibility constraint:

$$
\sum_{i \in S} x^{i}(\omega) \leq \sum_{i \in S} e^{i}(\omega) \text { for }(\mu-) \text { almost every } \omega \in \Omega .
$$

Given a subfield $\digamma^{\prime}$ of $\digamma$, we use the extended notion of $\digamma^{\prime}$-measurability by calling a function, which is equal $\mu$-almost everywhere to an $\digamma^{\prime}$-measurable function, also $\digamma^{\prime}$-measurable. A private $S$-allocation is an $S$-allocation $\mathbf{x}=\left(x^{i}\right)_{i \in S}$ such that $x^{i}$ is $\digamma^{i}$-measurable for every $i \in S$. An $N$-allocation (respectively, private $N$-allocation) is called an allocation (respectively, private allocation). We assume that the $N$-tuple of the initial endowments $\mathbf{e}=\left(e^{1}, \ldots, e^{n}\right)$ is a private allocation.

The preferences of agent $i$ over the commodity space are represented by a statedependent utility function, $u^{i}: \Omega \times R_{+}^{m} \rightarrow R_{+}$, measurable with respect to the product field $\digamma \times \mathcal{B}$ (where $\mathcal{B}$ is the $\sigma$-field of Borel sets in $R_{+}^{m}$ ) and continuous ${ }^{9}$.

\footnotetext{
7 Or, to be precise, their equivalence classes, where any two functions which are equal $\mu$-almost everywhere are identified.

8 For any two vectors $x=\left(x_{1}, \ldots, x_{m}\right), y=\left(y_{1}, \ldots, y_{m}\right) \in R^{m}$ we write $x \geq y$ when $x_{k} \geq y_{k}$ for every $k=1, \ldots, m$, and $x>y$ when $x \geq y$ and $x \neq y$.

9 Continuity of $u$ implies its $\digamma \times \mathcal{B}$-measurability when $\digamma$ is the $\sigma$-field of Borel sets in $\Omega$.
} 
We will always assume that $u^{i}(\omega, \cdot)$ is concave on $R_{+}^{m}$ for every $\omega \in \Omega$, and non-decreasing. If $\mathbf{x}=\left(x^{1}, \ldots, x^{n}\right)$ is an assignment, we denote

$$
U^{i}\left(x^{i}\right)=\int_{\Omega} u^{i}\left(\omega, x^{i}(\omega)\right) d \mu
$$

whenever the integral exists.

To sum up, an economy with differential information, $\mathcal{E}$, is described by a collection $\left(e^{i}, u^{i}, \digamma^{i}\right)_{i=1}^{n}$.

A possible interpretation ${ }^{10}$ of the above economy is the following. It extends over two periods of time. In the first period there is uncertainty about the state of nature. In this period, agents make contracts on redistribution of their initial endowments either before the state of nature is realized (ex-ante) or after receiving their private information (interim). In the second period agents carry out previously made agreements, and consumption takes place.

In order to define convergence of economies with differential information, we use a pseudo-metric (introduced in Boylan, 1971) on the family $\digamma^{*}$ of $\sigma$-subfields of $\digamma$, given by

$$
d\left(\digamma_{1}, \digamma_{2}\right)=\sup _{A \in \digamma_{1}} \inf _{B \in \digamma_{2}} \mu(A \triangle B)+\sup _{B \in \digamma_{2}} \inf _{A \in \digamma_{1}} \mu(A \triangle B),
$$

where $A \triangle B=(A \backslash B) \cup(B \backslash A)$ is the "symmetric difference" of $A$ and $B$. Consider a sequence $\left\{\mathcal{E}_{k}\right\}_{k=1}^{\infty}$ of economies $\mathcal{E}_{k}=\left(e_{k}^{i}, u_{k}^{i}, \digamma_{k}^{i}\right)_{i=1}^{n}$ with differential information. We say that $\left\{\mathcal{E}_{k}\right\}_{k=1}^{\infty}$ converges $^{11}$ to an economy $\mathcal{E}=\left(e^{i}, u^{i}, \digamma^{i}\right)_{i=1}^{n}$ if for every $i \in N$ :

i) ${ }^{12} e_{k}^{i} \rightarrow_{k \rightarrow \infty}^{L_{1}^{m}} e^{i}$;

ii) ${ }^{13} u_{k}^{i} \rightarrow_{k \rightarrow \infty} u^{i}$ uniformly on every compact subset of $\Omega \times R_{+}^{m}$;

iii) $\digamma_{k}^{i} \rightarrow_{k \rightarrow \infty} \digamma^{i}$ in Boylan pseudo-metric.

Finally, if $X$ is a random variable on $(\Omega, \digamma, \mu)$ and $\digamma^{\prime}$ is a $\sigma$-subfield of $\digamma$, denote by $E\left(X \mid \digamma^{\prime}\right)$ the conditional expectation ${ }^{14}$ of $X$ with respect to $\digamma^{\prime}$.

10 This paragraph is a quotation from Allen and Yannelis (2001), p. 265, slightly modified to fit our setting.

11 We use the language of "convergence of economies" only for the sake of convenience. As was mentioned in the introduction, the convergence here is actually restricted to the agents' characteristics.

12 By “ $\rightarrow \begin{gathered}L_{p}^{m} \\ k \rightarrow \infty\end{gathered}$ " we denote convergence in the $L_{p}^{m}$-norm.

13 Note that with this form of convergence of utility functions, the underlying preferences may fail to converge (for instance, when $u_{k}^{i}$ is defined as $\frac{1}{k} u$ for some utility function $u$, the sequence $\left\{u_{k}^{i}\right\}_{k=1}^{\infty}$ converges to $u^{i} \equiv 0$ ). However, this only makes our upper semi-continuity results (Theorems 1,2 , and 3 ) stronger, since there is no explicit assumption on convergence of the underlying preferences. And the discontinuity we show in Examples 1 and 2 is not due to this phenomenon: in both examples, utility functions of agents are fixed for all economies.

${ }^{14}$ Since $E\left(X \mid \digamma^{\prime}\right)$ is usually defined as a class of functions, we will always take a selection from it. 


\section{Continuity of Walrasian expectations equilibrium correspondence}

Radner $(1968,1982)$ introduced the notion of Walrasian expectations equilibrium (WEE) for economies with differential information. In this section we study continuity of the WEE correspondence.

In what follows we consider commodity bundles that are members of $L_{p}^{m}$, for some given $p \geq 1$. Let $\mathcal{E}=\left(e^{i}, u^{i}, \digamma^{i}\right)_{i=1}^{n}$ be an economy with differential information. A price system $\pi$ is a non-negative function in the unit sphere of $L_{q}^{m}$ (i.e., $\|\pi\|_{q}=1$ ), where $q \in(1, \infty]$ is such that $\frac{1}{p}+\frac{1}{q}=1$. It is elementary that any price functional $\varphi$ in the economy (which is a continuous linear functional of norm 1 on $L_{p}^{m}$, restricted to the cone of commodity bundles in $L_{p}^{m}$ ) is representable by a unique price system $\pi_{\varphi}$ : for any commodity bundle $x, \varphi(x)=\int_{\Omega} \pi_{\varphi} \cdot x d \mu$. Given a price system $\pi$, the budget set of $i \in N$ is given by

$$
\begin{aligned}
& B^{i}(\pi) \\
& =\left\{x \in L_{p}^{m} \mid x \text { is non-negative and } \digamma^{i} \text {-measurable and } \int_{\Omega} \pi \cdot x d \mu \leq \int_{\Omega} \pi \cdot e^{i} d \mu\right\} .
\end{aligned}
$$

A Walrasian expectations equilibrium (WEE) of $\mathcal{E}$ is a pair $(\mathbf{x}, \pi)$, where $\pi$ is a price system and $\mathbf{x}=\left(x^{1}, \ldots, x^{n}\right)$ is a private allocation such that for every $i \in N$ $x^{i}$ maximizes $U^{i}$ on $B^{i}(\pi)$. Denote by $W E E(\mathcal{E})$ the set of WEE in $\mathcal{E}$.

The following theorem (whose proof, together with all other proofs, is brought in Sect. 6) establishes upper semi-continuity of the WEE correspondence.

Theorem 1. Let $\left\{\mathcal{E}_{k}\right\}_{k=1}^{\infty}$ be a sequence of economies $\mathcal{E}_{k}=\left(e_{k}^{i}, u_{k}^{i}, \digamma_{k}^{i}\right)_{i=1}^{n}$ with differential information that converges to $\mathcal{E}=\left(e^{i}, u^{i}, \digamma^{i}\right)_{i=1}^{n}$, where $e^{i}$ is strictly positive for every $i \in N$. If $\left\{\left(\mathbf{x}_{k}, \pi_{k}\right)\right\}_{k=1}^{\infty}$ is a sequence such that $\left(\mathbf{x}_{k}, \pi_{k}\right) \in$ $W E E\left(\mathcal{E}_{k}\right)$ for every $k$, and for every $i \in N, x_{k}^{i} \rightarrow{ }_{k \rightarrow \infty}^{L_{p}^{m}} x^{i}, \pi_{k} \rightarrow{ }_{k \rightarrow \infty}^{L_{q}^{m}} \pi$, then $(\mathbf{x}, \pi) \in W E E(\mathcal{E})$.

One may ask whether the $L_{q}^{m}$-norm convergence of price systems can be replaced with the weak ${ }^{*}$ convergence ${ }^{15}$, without affecting the result. The answer is negative, as will be made clear in Example 1 of the next section. In this example we will also show that confining attention to WEE allocations only does not yield an upper semi-continuous correspondence.

\section{Continuity of the private core}

In this section we study the continuity of the private core of an economy with differential information, introduced in Yannelis (1991).

Let $\mathcal{E}=\left(e^{i}, u^{i}, \digamma^{i}\right)_{i=1}^{n}$ be an economy with differential information. The private core of $\mathcal{E}$ consists of all private allocations $\mathbf{x}=\left(x^{1}, \ldots, x^{n}\right)$ for which there do $n o t$ exist a non-empty coalition $S \subseteq N$ and a private $S$-allocation $\mathbf{y}=\left(y^{i}\right)_{i \in S}$, such that

$$
U^{i}\left(y^{i}\right)>U^{i}\left(x^{i}\right)
$$

15 When $1<p$, the weak* convergence is of course equivalent to the weak convergence. 
for every $i \in S$.

As was said in the introduction, in complete information economies the core correspondence is upper semi-continuous (see, e.g., Kannai, 1970). Therefore, it is natural to ask whether upper semi-continuity holds for economies with differential information. That is, if $\left\{\mathbf{x}_{k}\right\}_{k=1}^{\infty}$ is a sequence of private core allocations in a converging sequence of economies $\left\{\mathcal{E}_{k}\right\}_{k=1}^{\infty}$, and $\left\{\mathbf{x}_{k}\right\}_{k=1}^{\infty}$ converges to $\mathbf{x}$, is $\mathbf{x}$ a private core allocation of the limiting economy $\mathcal{E}$ ? The following example shows that the answer may be negative for all converging sequences $\left\{\mathbf{x}_{k}\right\}_{k=1}^{\infty}$, even in very simple economies.

Example 1. For every $k=0,1,2, \ldots$ let $\mathcal{E}_{k}$ be an economy in which $m=1$, $n=2, e_{k}^{1}=e_{k}^{2} \equiv \frac{1}{2}, \Omega=[0,1] \cup[2,3], \mu$ is the restriction of the Lebesgue measure on the real line to $\Omega$ (normalized so as to satisfy $\mu(\Omega)=1$ ), $u_{k}^{1}(\omega, x)=$ $\left\{\begin{array}{l}x, \text { if } \omega \in[0,1], \\ 0, \text { if } \omega \in[2,3] ;\end{array} u_{k}^{2}(\omega, x)=\left\{\begin{array}{l}0, \text { if } \omega \in[0,1], \\ x, \text { if } \omega \in[2,3] .\end{array}\right.\right.$ For $k>0$, private information fields of agents are given as follows: $\digamma_{k}^{1}$ is the finite field generated by $[0,1] \cup\left[2,2+\frac{1}{2 k}\right],\left(2+\frac{1}{2 k}, 3\right]$, and $\digamma_{k}^{2}$ is the finite field generated by $[0,1],[2,3]$. For $k=0, \digamma^{1}=\digamma^{2}$ is the finite field generated by $[0,1],[2,3]$.

Let $\mathbf{x}_{k}=\left(x_{k}^{1}, x_{k}^{2}\right)$ be a private core allocation ${ }^{16}$ in the economy $\mathcal{E}_{k}$. Since $\mathbf{x}_{k}$ is private, it has the form

$$
\mathbf{x}_{k}=\left(a_{1}(k) \chi_{[0,1] \cup\left[2,2+\frac{1}{2 k}\right]}+a_{2}(k) \chi_{\left(2+\frac{1}{2 k}, 3\right]}, b_{1}(k) \chi_{[0,1]}+b_{2}(k) \chi_{[2,3]}\right)
$$

(where $\chi_{A}$ stands for the indicator function of the set $A$ ); the equality holds almost everywhere. The feasibility constraint (1) taken for $\omega \in[0,1]$ and $\omega \in\left[2,2+\frac{1}{2 k}\right]$ (with $S=N$ ) yields

$$
a_{1}(k)+b_{1}(k) \leq 1, \text { and } a_{1}(k)+b_{2}(k) \leq 1 .
$$

Note that

$$
a_{1}(k) \geq \frac{1}{2}
$$

since, otherwise, for $S=\{1\}$ the $S$-allocation $\mathbf{y}=\left(e_{k}^{1}\right)$ satisfies

$$
U^{1}\left(y^{1}\right)>U^{1}\left(x_{k}^{1}\right),
$$

contrary to $\mathbf{x}_{k}$ being a private core allocation in the economy $\mathcal{E}_{k}$ (that is, $\mathbf{x}_{k}$ is not individually rational). Individual rationality of $\mathbf{x}_{k}$ also implies

$$
b_{2}(k) \geq \frac{1}{2}
$$

From (5), (6), and (4) we deduce that

$$
a_{1}(k)=b_{2}(k)=\frac{1}{2} .
$$

16 Existence of a private core allocation in such an economy can be established by using arguments of Glycopantis, Muir and Yannelis (2001) (where existence was established with the "no free disposal" condition). Alternatively, one can show nonemptiness of the private core of $\mathcal{E}_{\varepsilon}$ by checking that it contains the initial endowments allocation. 
Consider now a converging subsequence of $\left\{\left(a_{1}(k), a_{2}(k), b_{1}(k), b_{2}(k)\right)\right\}_{k=1}^{\infty}$. It exists since $0 \leq a_{1}(k), a_{2}(k), b_{1}(k), b_{2}(k) \leq 1$ by the feasibility constraint, and w.l.o.g. we will assume that the sequence itself converges. This implies convergence of $\left\{\mathbf{x}_{k}\right\}_{k=1}^{\infty}$ pointwise almost everywhere (and thus in $L_{1}^{1}$-norm, or more generally any $L_{p}^{1}$-norm for $1 \leq p<\infty$ due to the boundedness of the sequence) to

$$
\mathbf{x}=\left(a_{1} \chi_{[0,1]}+a_{2} \chi_{[2,3]}, b_{1} \chi_{[0,1]}+b_{2} \chi_{[2,3]}\right),
$$

with $a_{1}=b_{2}=\frac{1}{2}$. Also, $\left\{\mathcal{E}_{k}\right\}_{k=1}^{\infty}$ converges to $\mathcal{E}_{0}$. However, $\mathbf{x}$ is not a private core allocation of $\mathcal{E}_{0}$. Indeed, take $S=N$ and $\mathbf{y}=\left(\chi_{[0,1]}, \chi_{[2,3]}\right)$. This $\mathbf{y}$ is obviously a private allocation in $\mathcal{E}_{0}$, and

$$
U^{i}\left(y^{i}\right)>U^{i}\left(x^{i}\right)
$$

is satisfied for every $i$ (that is, $\mathbf{x}$ is not Pareto-optimal).

Note that this example would still work if the utility functions were slightly modified to become strictly concave and strictly increasing. The compact set $\Omega$ could also be made connected without affecting our claim.

Our final observation is that there is a sequence of WEE in $\left\{\mathcal{E}_{k}\right\}_{k=1}^{\infty}$ that does not converge to a WEE in $\mathcal{E}_{0}$. Indeed, the "no trade" scenario $\left(\mathbf{e}, \pi_{k}\right)$, where $\mathbf{e}$ consists of the initial endowments of the agents and $\pi_{k}$ (viewed as a function in $L_{q}^{1}$ ) is given by

$$
\pi_{k}=\left\{\begin{array}{cc}
(4 k)^{\frac{1}{q}} \chi_{\left[2,2+\frac{1}{2 k}\right]}, & \text { if } q<\infty, \\
\chi_{\left[2,2+\frac{1}{2 k}\right]}, & \text { if } q=\infty
\end{array},\right.
$$

constitutes a WEE in $\mathcal{E}_{k}$. However, $\left\{\pi_{k}\right\}_{k=1}^{\infty}$ clearly does not converge in the $L_{q}^{1}$ norm. It does converge weakly*, but to the zero function. This shows that Theorem 1 cannot be strengthened by assuming that $\left\{\pi_{k}\right\}_{k=1}^{\infty}$ converges to $\pi$ weakly* (since $\pi$ can be zero despite that $\left\|\pi_{k}\right\|_{q}=1$ for every $k$ ).

In the limiting economy $\mathcal{E}_{0}$, every WEE has the form $(\mathbf{y}, \pi)$, where $\mathbf{y}=$ $\left(\chi_{[0,1]}, \chi_{[2,3]}\right)$ (almost everywhere) and $\int_{[0,1]} \pi(\omega) d \mu(\omega)=\int_{[2,3]} \pi(\omega) d \mu(\omega)$. This shows that the set of WEE allocations $W E E A$ is also not upper semicontinuous: $\mathbf{e} \in W E E A\left(\mathcal{E}_{k}\right)$ for every $k$, but $W E E A\left(\mathcal{E}_{0}\right)=\{\mathbf{y}\}$ and $\mathbf{y} \neq \mathbf{e}$.

In the above example the limiting information field is neither included, nor includes, the information fields that converge to it. However, when this does not occur, positive results on upper semi-continuity of the private core can be obtained.

Theorem 2. Let $\left\{\mathcal{E}_{k}\right\}_{k=1}^{\infty}=\left\{\left(e_{k}^{i}, u_{k}^{i}, \digamma_{k}^{i}\right)_{i=1}^{n}\right\}_{k=1}^{\infty}$ be a sequence of economies with differential information that converges ${ }^{17}$ to $\mathcal{E}=\left(e^{i}, u^{i}, \digamma^{i}\right)_{i=1}^{n}$, such that $\digamma_{k}^{i} \supseteq \digamma^{i}$ for all $i \in N$ and $k$. If $\left\{\mathbf{x}_{k}\right\}_{k=1}^{\infty}$ is such that $\mathbf{x}_{k}=\left(x_{k}^{1}, \ldots, x_{k}^{n}\right)$ is a private core allocation in $\mathcal{E}_{k}$, and for every $i \in N x_{k}^{i} \rightarrow \rightarrow_{k \rightarrow \infty}^{L_{1}^{m}} x^{i}$, then $\mathbf{x}=\left(x^{1}, \ldots, x^{n}\right)$ is a private core allocation in $\mathcal{E}$.

17 Convergence of information fields in Boylan pseudo-metric (which is one of the aspects of convergence of economies) is not, in fact, necessary for this theorem. 
According to Theorem 2, if the information fields of agents shrink when they approach the limiting information fields (which can be the case when, e.g., the level of random noise increases in the limit), then the private core is upper semicontinuous. Theorem 3 below may be viewed as a dual result, showing that the private core is also upper semi-continuous when the information of agents expands to the fullest possible extent, i.e., when the economies converge to a complete information economy. As was said in the Introduction, it is related to the result of Balder and Yannelis (2002), who established continuity of the private core correspondence in more general economies, but when information endowments are increasing monotonically. At the same time, Theorem 3 stands in contrast to the result of Krasa and Shafer (2001), that shows generic discontinuity of the private core correspondence when the complete information is approached by changing the common prior belief of agents (rather than by expanding their information fields).

Theorem 3. Let $\left\{\mathcal{E}_{k}\right\}_{k=1}^{\infty}=\left\{\left(e_{k}^{i}, u_{k}^{i}, \digamma_{k}^{i}\right)_{i=1}^{n}\right\}_{k=1}^{\infty}$ be a sequence of economies with differential information that converges to $\mathcal{E}=\left(e^{i}, u^{i}, \digamma^{i}\right)_{i=1}^{n}$, where $\digamma^{i}=\digamma$ for every $i \in N, \digamma$ is the $\sigma$-field of all Borel sets in $\Omega$, and each $e^{i}$ is a continuous and strictly positive function. Assume also that for every two disjoint and closed subsets $A$ and $B$ of $\Omega$ there exist $A_{k}^{i} \in \digamma_{k}^{i}$ for every $i$ and $k$ such that $A \subseteq A_{k}^{i}$ and $A_{k}^{i} \cap B=\emptyset$ for all sufficiently large $k$ (that is, $\digamma_{k}^{i}$ can separate disjoint closed sets for all sufficiently large $k$ ). Then, if $\left\{\mathbf{x}_{k}\right\}_{k=1}^{\infty}$ is such that $\mathbf{x}_{k}=\left(x_{k}^{1}, \ldots, x_{k}^{n}\right)$ is a private core allocation in $\mathcal{E}_{k}$ and for every $i \in N x_{k}^{i} \rightarrow_{k \rightarrow \infty}^{L_{1}^{m}} x^{i}, \mathbf{x}=\left(x^{1}, \ldots, x^{n}\right)$ is a private core allocation in $\mathcal{E}$.

\section{The coarse core}

Let $\mathcal{E}=\left(e^{i}, u^{i}, \digamma^{i}\right)_{i=1}^{n}$ be an economy with differential information. The coarse core of $\mathcal{E}$ consists of all allocations $\mathbf{x}=\left(x^{1}, \ldots, x^{n}\right)$ for which there do not exist a non-empty coalition $S \subseteq N$, an event $A \in \bigwedge_{i \in S} \digamma^{i}$ with $\mu(A)>0$ (where $\bigwedge_{i \in S} \digamma^{i}$ denotes the intersection of all $\digamma^{i}, i \in S$, and represents the common knowledge of agents in $S$ ), and an $S$-assignment $\mathbf{y}=\left(y^{i}\right)_{i \in S}$, that satisfy the following conditions:

(i) $\sum_{i \in S} y^{i}(\omega) \leq \sum_{i \in S} e^{i}(\omega)$ for almost every $\omega \in A$;

(ii) $^{18} E\left(u^{i}\left(\cdot, y^{i}(\cdot)\right) \mid \digamma^{i}\right)(\omega)>E\left(u^{i}\left(\cdot, x^{i}(\cdot)\right) \mid \digamma^{i}\right)(\omega)$ for almost every $\omega \in A$ and $i \in S$.

According to (i), y is feasible for agents in $S$, given the event $A$ and their initial endowments. And (ii) means that agents in $S$ can improve their conditional expected utility when they redistribute their endowments according to $\mathbf{y}$, given that $A$ occurred. The concept of coarse core (introduced by Wilson, 1978) thus disallows existence of such $S, A$, and $\mathbf{y}$.

Wilson (1978) proved that the coarse core is non-empty under standard conditions on the economy, at least when the set of states of nature is finite. The following

18 Recall that $E\left(X \mid \digamma^{\prime}\right)$ stands for the conditional expectation of $X$ with respect to $\digamma^{\prime}$. 
example shows that the coarse core may not be upper semi-continuous, even when the private core is ${ }^{19}$.

Example 2. Consider a sequence of economies $\mathcal{E}_{k}=\left(e_{k}^{i}, u_{k}^{i}, \digamma_{k}^{i}\right)_{i=1}^{n}$ with $m=2$ and $n=4$, where:

1) $u_{k}^{i}$ is state-independent, and $u_{k}^{i}\left(x_{1}, x_{2}\right)=u\left(x_{1}, x_{2}\right)=\left(\sqrt{x_{1}}+\sqrt{x_{2}}\right)^{2}$ for every $i, k$;

2) $e_{k}^{i}$ is state-independent, and $e_{k}^{1}=e_{k}^{3}=(10,110), e_{k}^{2}=e_{k}^{4}=(110,10)$, for every $k$;

3 ) the compact space of states $\Omega$ is the sequence $\left\{\omega_{j}\right\}_{j=0}^{\infty}$, where $\omega_{0}=0$, and $\omega_{j}=\frac{1}{j}$ for $j \geq 1$;

4) $\mu$ is a measure on $\Omega$ according to which $\mu\left(\left\{\omega_{2 j}\right\}\right)=\mu\left(\left\{\omega_{2 j+1}\right\}\right)>0$ for every $j \geq 0$;

5) $\digamma_{k}^{1}=\digamma_{k}^{2}=\left\{\emptyset,\left\{\omega_{2 j}\right\}_{j=0}^{\infty},\left\{\omega_{2 j+1}\right\}_{j=0}^{\infty}, \Omega\right\}$; that is, 1 and 2 can only tell whether the realized state of nature has even or odd index;

6) $\digamma_{k}^{3}=\digamma_{k}^{4}$ is the minimal $\sigma$-field in $\Omega$ that contains $\left\{\omega_{0}\right\},\left\{\omega_{1}\right\}, \ldots,\left\{\omega_{k}\right\}$, and $\left\{\omega_{j}\right\}_{j=k}^{\infty}$; that is, 3 and 4 can actually know the state of nature, provided its index is less than $k$.

It is clear that the sequence $\left\{\mathcal{E}_{k}\right\}_{k=1}^{\infty}$ converges to an economy $\mathcal{E}=$ $\left(e^{i}, u^{i}, \digamma^{i}\right)_{i=1}^{n}$, which differs from economies in $\left\{\mathcal{E}_{k}\right\}_{k=1}^{\infty}$ only in the following respect: $\digamma^{3}=\digamma^{4}$ is the field of all subsets of $\Omega$.

Now consider an assignment $\mathbf{x}=\left(x^{1}, x^{2}, x^{3}, x^{4}\right)$, where $x^{1}\left(\omega_{2 j}\right)=$ $x^{2}\left(\omega_{2 j+1}\right)=(50,50)$ and $x^{1}\left(\omega_{2 j+1}\right)=x^{2}\left(\omega_{2 j}\right)=(70,70)$ for every $j \geq 0$, and $x^{3}=x^{4} \equiv(60,60)$. It is obvious that $\mathrm{x}$ is an allocation for all economies in $\left\{\mathcal{E}_{k}\right\}_{k=1}^{\infty}$, and for $\mathcal{E}$.

We show first that $\mathbf{x}$ is not a coarse core allocation for $\mathcal{E}$. Indeed, $S=\{2,3\}$ can improve the conditional expected utility of both 2 and 3 given that the state of nature has an odd index. We simply take $A=\left\{\omega_{2 j+1}\right\}_{j=0}^{\infty}$ (which is clearly in the common knowledge of 2 and 3 in $\mathcal{E}: A \in \digamma^{2} \wedge \digamma^{3}$ ), and $\mathbf{y}=\left(y^{i}\right)_{i \in S}$ defined by $y^{2}\left(\omega_{2 j+1}\right) \equiv(51,51), y^{3}\left(\omega_{2 j+1}\right) \equiv(69,69)$. Then (i) and (ii) are satisfied, and hence $\mathrm{x}$ is not a coarse core allocation for $\mathcal{E}$.

Our next step is to show that $\mathrm{x}$ is a coarse core allocation for every $\mathcal{E}_{k}$. (This will imply that the coarse core correspondence indeed lacks upper semi-continuity.) To this end suppose that there are $k, S, A$, and y that satisfy (i) and (ii) in the economy $\mathcal{E}_{k}$. It is easy to see that $\mathrm{x}$ is individually rational (given each trader's information), and so $S$ must contain more than one trader.

19 In this example, information fields of the first two agents are fixed in the converging sequence of economies, while for the other (last) two agents the information fields approximate full infomation. The proof of Theorem 3 can be used to show that the limit of any converging sequence of private core allocations will be a private core allocation in the limiting economy (commodity bundles $y^{1}$ and $y^{2}$, if used by a blocking coalition in $\mathcal{E}$, will not even have to be approximated by a continuous function in order to show that $E\left(y^{1} \mid \digamma_{k}^{1}\right)$ and $E\left(y^{2} \mid \digamma_{k}^{2}\right)$ approximate them uniformly). 
Note that (ii) implies

$$
\begin{aligned}
& \int_{A} u\left(y^{i}(\omega)\right) d \mu(\omega)=\int_{A} E\left(u\left(y^{i}(\cdot)\right) \mid \digamma^{i}\right)(\omega) d \mu(\omega) \\
& >\int_{A} E\left(u\left(x^{i}(\cdot)\right) \mid \digamma^{i}\right)(\omega) d \mu(\omega)=\int_{A} u\left(x^{i}(\omega)\right) d \mu(\omega)
\end{aligned}
$$

for every $i \in S$, since $A$ is an element of every $F_{i}$. Therefore

$$
\sum_{i \in S} \int_{A} u\left(y^{i}(\omega)\right) d \mu(\omega)>\sum_{i \in S} \int_{A} u\left(x^{i}(\omega)\right) d \mu(\omega) .
$$

At the same time, from concavity and homogeneity of $u$ and (i) it follows that:

$$
\begin{aligned}
& \sum_{i \in S} \int_{A} u\left(y^{i}(\omega)\right) d \mu(\omega) \leq u\left(\sum_{i \in S} \int_{A} y^{i}(\omega) d \mu(\omega)\right) \\
& =\left(\sqrt{\sum_{i \in S} \int_{A} y^{i}(\omega)_{1} d \mu(\omega)}+\sqrt{\sum_{i \in S} \int_{A} y^{i}(\omega)_{2} d \mu(\omega)}\right)^{2} \\
& \leq\left(2 \sqrt{\frac{\sum_{i \in S} \int_{A}\left(y^{i}(\omega)_{1}+y^{i}(\omega)_{2}\right) d \mu(\omega)}{2}}\right)^{2} \\
& =2 \int_{A} \sum_{i \in S}\left(y^{i}(\omega)_{1}+y^{i}(\omega)_{2}\right) d \mu(\omega) \\
& \leq 2 \int_{A}\left(e_{i \in S}(\omega)_{1}+e^{i}(\omega)_{2}\right) d \mu(\omega)=2 \int_{A} 120|S| d \mu(\omega) \\
& =240|S| \mu(A) .
\end{aligned}
$$

Thus

$$
\sum_{i \in S} \int_{A} u\left(y^{i}(\omega)\right) d \mu(\omega) \leq 240|S| \mu(A) .
$$

If $S=\{1,2\}$ or $S=\{3,4\}$, then for each $\omega \sum_{i \in S} u\left(x^{i}(\omega)\right)=480=240|S|$, and so (7) and (8) are inconsistent. Since $|S|>1$ (as was mentioned), we are left with the possibility that $S \cap\{1,2\} \neq \emptyset$ and $S \cap\{3,4\} \neq \emptyset$. Then $A=\Omega$ since the common knowledge of all the agents in $S$ (where $A$ belongs) contains only trivial information ${ }^{20}$, and $\mu(A)>0$. However, clearly $\int_{\Omega} u\left(x^{i}(\omega)\right) d \mu(\omega)=240$ for every $i \in N$, and therefore

$$
\sum_{i \in S} \int_{\Omega} u\left(x^{i}(\omega)\right) d \mu(\omega)=240|S|
$$

Thus, (7) and (8) lead to a contradiction again. We conclude that there exist no $k$, $S, A$, and $\mathbf{y}$ that satisfy (i) and (ii) in the economy $\mathcal{E}_{k}$, and therefore $\mathrm{x}$ is a coarse core allocation in every $\mathcal{E}_{k}$.

20 I.e., the field $\{\emptyset, \Omega\}$. 


\section{Proofs}

Lemma 1. Let $\left\{\digamma_{k}\right\}_{k=1}^{\infty}$ be a sequence of $\sigma$-subfields of $\digamma$ that converges to $\digamma_{0}$ in Boylan pseudo-metric, and let $\left\{X_{k}\right\}_{k=1}^{\infty}$ be a sequence of functions in $L_{1}(\Omega, \digamma, \mu)$ that converges to $X$ in $L_{1}$-norm. If $X_{k}$ is $\digamma_{k}$-measurable for every $k$, then $X$ is $\digamma_{0}$-measurable.

Proof. We will show that $X=E\left(X \mid \digamma_{0}\right)$ almost everywhere. Since $L_{1}$-norm convergence implies convergence in measure, $\left\{X_{k}\right\}_{k=1}^{\infty}$ converges to $X$ in measure. Thus, it suffices to show that $\left\{X_{k}\right\}_{k=1}^{\infty}$ converges in measure also to $E\left(X \mid \digamma_{0}\right)$.

From $\digamma_{k}$-measurability of $X_{k}, X_{k}=E\left(X_{k} \mid \digamma_{k}\right)$ almost everywhere. Thus, almost everywhere,

$$
\begin{aligned}
& \left|X_{k}-E\left(X \mid \digamma_{0}\right)\right|=\left|E\left(X_{k} \mid \digamma_{k}\right)-E\left(X \mid \digamma_{0}\right)\right| \\
& \leq\left|E\left(X_{k} \mid \digamma_{k}\right)-E\left(X \mid \digamma_{k}\right)\right|+\left|E\left(X \mid \digamma_{k}\right)-E\left(X \mid \digamma_{0}\right)\right| .
\end{aligned}
$$

For every $k$,

$$
\begin{aligned}
& \int_{\Omega}\left|E\left(X_{k} \mid \digamma_{k}\right)-E\left(X \mid \digamma_{k}\right)\right| d \mu \leq \int_{\Omega} E\left(\left|X_{k}-X\right| \mid \digamma_{k}\right) d \mu \\
& =\int_{\Omega}\left|X_{k}(\omega)-X(\omega)\right| d \mu(\omega)=\left\|X_{k}-X\right\|_{1} .
\end{aligned}
$$

Since $\left\|X_{k}-X\right\|_{1} \rightarrow_{k \rightarrow \infty} 0$, also $\int_{\Omega}\left|E\left(X_{k} \mid \digamma_{k}\right)-E\left(X \mid \digamma_{k}\right)\right| d \mu \rightarrow_{k \rightarrow \infty} 0$, and hence the first summand in (10) converges to zero in measure. And $E(X$ $\left.\digamma_{k}\right) \rightarrow_{k \rightarrow \infty} E\left(X \mid \digamma_{0}\right)$ in measure by Theorem 4 in Boylan (1971). Consequently, by (9)-(10), $\left\{X_{k}\right\}_{k=1}^{\infty}$ indeed converges in measure to $E\left(X \mid \digamma_{0}\right)$.

Lemma 2. Let $\left\{u_{k}\right\}_{k=1}^{\infty}$ be a sequence of continuous functions on $\Omega \times R_{+}^{m}$ such that for every $\omega \in \Omega$ the function $u_{k}(\omega, \cdot)$ is concave, non-negative, and nondecreasing $^{21}$. Assume that $\left\{u_{k}\right\}_{k=1}^{\infty}$ converges to a function $u$ uniformly on every compact subset of $\Omega \times R_{+}^{m}$. If $\left\{x_{k}\right\}_{k=1}^{\infty}$ is a sequence in $L_{1}^{m}$ that converges to $x$ in the $L_{1}^{m}$-norm, then

$$
\lim _{k \rightarrow \infty} \int_{\Omega} u_{k}\left(\omega, x_{k}(\omega)\right) d \mu(\omega)=\int_{\Omega} u(\omega, x(\omega)) d \mu(\omega) .
$$

Proof. Due to the uniform convergence of $\left\{u_{k}\right\}_{k=1}^{\infty}$, the sequence of $C_{k}=$ $\max _{\omega \in \Omega,\|x\| \leq 1} u_{k}(\omega, x)$ is bounded from above by some $C>0$. Fix $\varepsilon>0$. Since $\left\{x_{k}\right\}_{k=1}^{\infty}$ converges to $x$ in $L_{1}^{m}$-norm, $\left\{x_{k}\right\}_{k=1}^{\infty}$ is uniformly integrable (see Proposition II.5.4 of Neveu, 1965) and $x$ is integrable. Consequently, we can find $M>1$ with:

$$
\text { (i) } \sup _{k} \int_{\left\{\omega \in \Omega \mid\left\|x_{k}(\omega)\right\|>M\right\}}\left\|x_{k}(\omega)\right\| d \mu(\omega)<\frac{\varepsilon}{9 C} ;
$$

${ }^{21}$ As will become evident in the proof, these three requirements could be replaced by the following (weaker) condition: $\left|u_{k}(\omega, x)\right| \leq C_{k} \max \{\|x\|, 1\}$ for all $k, \omega \in \Omega, x \in R_{+}^{l}$, and some constant $C_{k}$ such that $\lim \sup _{k \rightarrow \infty} C_{k}<\infty$. 
and

$$
\text { (ii) } \int_{\{\omega \in \Omega \mid\|x(\omega)\|>M\}}\|x(\omega)\| d \mu(\omega)<\frac{\varepsilon}{9 C} \text {. }
$$

Note that concavity and monotonicity of each $u_{k}(\omega, \cdot)$, and the convergence of $\left\{u_{k}\right\}_{k=1}^{\infty}$ to $u$, imply that

$$
u(\omega, x), u_{k}(\omega, x) \leq C \max \{\|x\|, 1\}
$$

for all $k, \omega \in \Omega$, and $x \in R_{+}^{l}$. In particular, the integral $\int_{\Omega} u(\omega, x(\omega)) d \mu(\omega)$ is well defined.

Now,

$$
\begin{aligned}
& \left|\int_{\Omega} u_{k}\left(\omega, x_{k}(\omega)\right) d \mu(\omega)-\int_{\Omega} u(\omega, x(\omega)) d \mu(\omega)\right| \\
& \leq \int_{\Omega}\left|u_{k}\left(\omega, x_{k}(\omega)\right)-u\left(\omega, x_{k}(\omega)\right)\right| d \mu(\omega) \\
& \quad+\int_{\Omega}\left|u\left(\omega, x_{k}(\omega)\right)-u(\omega, x(\omega))\right| d \mu(\omega) \\
& =\int_{\Omega}\left|u_{k}\left(\omega, x_{k}(\omega)\right)-u\left(\omega, x_{k}(\omega)\right)\right| \chi_{\left\{\omega \in \Omega \mid\left\|x_{k}(\omega)\right\| \leq M\right\}} d \mu(\omega) \\
& \quad+\int_{\left\{\omega \in \Omega \mid\left\|x_{k}(\omega)\right\|>M\right\}}\left|u_{k}\left(\omega, x_{k}(\omega)\right)-u\left(\omega, x_{k}(\omega)\right)\right| d \mu(\omega) \\
& \quad+\int_{\Omega}\left|u\left(\omega, x_{k}(\omega)\right)-u(\omega, x(\omega))\right| \chi_{\left\{\omega \in \Omega \mid\left\|x_{k}(\omega)\right\| \leq M \text { and }\|x(\omega)\| \leq M\right\}} d \mu(\omega) \\
& \quad+\int_{\left\{\omega \in \Omega \mid\left\|x_{k}(\omega)\right\|>M \text { or }\|x(\omega)\|>M\right\}}\left|u\left(\omega, x_{k}(\omega)\right)-u(\omega, x(\omega))\right| d \mu(\omega) \\
& \equiv I_{1}+I_{2}+I_{3}+I_{4} .
\end{aligned}
$$

(Here $\chi_{S}$ stands for the characteristic function of the set $S$ ). We will show that each $I_{j}$ is less than $\varepsilon$ for all sufficiently large $k$, and this will prove the lemma.

The uniform convergence of $\left\{u_{k}\right\}_{k=1}^{\infty}$ to $u$ on $\Omega \times\left\{x \in R_{+}^{m} \mid\|x\| \leq\right.$ $M\}$ immediately yields $\lim _{k \rightarrow \infty} I_{1}=0$, and thus

$$
I_{1}<\varepsilon
$$

for all sufficiently large $k$. Inequality (11) implies that

$$
I_{2} \leq 2 C \int_{\left\{\omega \in \Omega \mid\left\|x_{k}(\omega)\right\|>M\right\}}\left\|x_{k}(\omega)\right\| d \mu(\omega),
$$

and by our choice of $M$,

$$
I_{2}<\varepsilon
$$

for all $k$. Since $x_{k}$ converges to $x$ in measure (as implied by the $L_{1}^{m}$-norm convergence), and $u(\cdot, \cdot)$ is uniformly continuous on the compact set $\Omega \times$ 
$\left\{x \in R_{+}^{m} \mid\|x\| \leq M\right\}$ (being a continuous function), $\mid u\left(\omega, x_{k}(\omega)\right)-$ $u(\omega, x(\omega)) \mid \cdot \chi_{\left\{\omega \in \Omega \mid\left\|x_{k}(\omega)\right\| \leq M \text { and }\|x(\omega)\| \leq M\right\}}$ converges in measure to zero, and thus $\lim _{k \rightarrow \infty} I_{3}=0$ by the generalized bounded convergence theorem. Once again,

$$
I_{3}<\varepsilon
$$

for all sufficiently large $k$. Finally,

$$
\begin{aligned}
I_{4}= & \int_{\left\{\omega \in \Omega \mid\left\|x_{k}(\omega)\right\|>M \text { or }\|x(\omega)\|>M\right\}}\left|u\left(\omega, x_{k}(\omega)\right)-u(\omega, x(\omega))\right| d \mu(\omega) \\
\leq & \int_{\left\{\omega \in \Omega \mid\left\|x_{k}(\omega)\right\|>M\right\}}\left|u\left(\omega, x_{k}(\omega)\right)-u(\omega, x(\omega))\right| d \mu(\omega) \\
& +\int_{\{\omega \in \Omega \mid\|x(\omega)\|>M\}}\left|u\left(\omega, x_{k}(\omega)\right)-u(\omega, x(\omega))\right| d \mu(\omega) \\
\leq & C \int_{\left\{\omega \in \Omega \mid\left\|x_{k}(\omega)\right\|>M\right\}}\left(\left\|x_{k}(\omega)\right\|+\max (\|x(\omega)\|, 1)\right) d \mu(\omega) \\
& +C \int_{\{\omega \in \Omega \mid\|x(\omega)\|>M\}}\left(\max \left(\left\|x_{k}(\omega)\right\|, 1\right)+\|x(\omega)\|\right) d \mu(\omega) \\
\leq & C \int_{\left\{\omega \in \Omega \mid\left\|x_{k}(\omega)\right\|>M\right\}}\left(2\left\|x_{k}(\omega)\right\|+\|x(\omega)\|\right) d \mu(\omega) \\
& +C \int_{\{\omega \in \Omega \mid\|x(\omega)\|>M\}}\left(\left\|x_{k}(\omega)\right\|+2\|x(\omega)\|\right) d \mu(\omega) \\
\leq & C \int_{\left\{\omega \in \Omega \mid\left\|x_{k}(\omega)\right\|>M\right\}}\left(2\left\|x_{k}(\omega)\right\|+\left\|x(\omega)-x_{k}(\omega)\right\|+\left\|x_{k}(\omega)\right\|\right) d \mu(\omega) \\
& +C \int_{\{\omega \in \Omega \mid\|x(\omega)\|>M\}}\left(\left\|x_{k}(\omega)-x(\omega)\right\|+\|x(\omega)\|+2\|x(\omega)\|\right) d \mu(\omega) \\
\leq & 2 C \int_{\Omega}\left\|x_{k}(\omega)-x_{0}(\omega)\right\| d \mu(\omega) \\
& +3 C \int_{\left\{\omega \in \Omega \mid\left\|x_{k}(\omega)\right\|>M\right\}}\left\|x_{k}(\omega)\right\| d \mu(\omega)+3 C \int_{\left\{\omega \in \Omega \mid\left\|x_{0}(\omega)\right\|>M\right\}}\left\|x_{0}(\omega)\right\| d \mu(\omega) \\
\leq & 2 C \int_{\Omega}\left\|x_{k}(\omega)-x_{0}(\omega)\right\| d \mu(\omega)+\frac{2 \varepsilon}{3}(\text { by the choice of } M) .
\end{aligned}
$$

The first summand in the above expression is less than $\frac{\varepsilon}{3}$ for all sufficiently large $k$, which yields

$$
I_{4}<\varepsilon
$$

Proof of Theorem 1. We have to show that $(\mathbf{x}, \pi)$ is a WEE of $\mathcal{E}$. Note first that $\mathbf{x}=\left(x^{1}, \ldots, x^{n}\right)$ is a private allocation in $\mathcal{E}$. Indeed, each $x^{i}$ is $\digamma^{i}$-measurable by Lemma 1. Moreover, each $L_{1}^{m}$-norm convergent $\left\{x_{k}^{i}\right\}_{k=1}^{\infty}$ has a subsequence that converges pointwise almost everywhere, and thus $\mathbf{x}$ also satisfies the feasibility constraint (1) (for $S=N$ ). 
Next, for every $i \in N$

$$
\left|\int_{\Omega}\left(\pi_{k} \cdot x_{k}^{i}-\pi \cdot x^{i}\right) d \mu\right| \leq \int_{\Omega}\left|\pi_{k} \cdot\left(x_{k}^{i}-x^{i}\right)\right| d \mu+\int_{\Omega}\left|\left(\pi_{k}-\pi\right) \cdot x^{i}\right| d \mu .
$$

By Hölder inequality,

$$
\int_{\Omega}\left|\pi_{k} \cdot\left(x_{k}^{i}-x^{i}\right)\right| d \mu \leq\left\|\pi_{k}\right\|_{q}\left\|x_{k}^{i}-x^{i}\right\|_{p}=\left\|x_{k}^{i}-x^{i}\right\|_{p}
$$

and

$$
\int_{\Omega}\left|\left(\pi_{k}-\pi\right) \cdot x^{i}\right| d \mu \leq\left\|\pi_{k}-\pi\right\|_{q}\left\|x^{i}\right\|_{p}
$$

However, $\lim _{k \rightarrow \infty}\left\|\pi_{k}-\pi\right\|_{q}=\lim _{k \rightarrow \infty}\left\|x_{k}^{i}-x^{i}\right\|_{p}=0$, and hence by (13)

$$
\int_{\Omega} \pi_{k} \cdot x_{k}^{i} d \mu \rightarrow_{k \rightarrow \infty} \int_{\Omega} \pi \cdot x^{i} d \mu
$$

It is also obvious that

$$
\int_{\Omega} \pi_{k} \cdot e^{i} d \mu \rightarrow_{k \rightarrow \infty} \int_{\Omega} \pi \cdot e^{i} d \mu
$$

From (14), (15) it follows that $x^{i} \in B^{i}(\pi)$ for every $i \in N$.

It remains to show that for every $i \in N, x^{i}$ maximizes $U^{i}$ on $B^{i}(\pi)$. Indeed, if this were not true, there would exist $i \in N$ and $y^{i} \in B^{i}(\pi)$ with $U^{i}\left(y^{i}\right)>U^{i}\left(x^{i}\right)$. Since $(1-\alpha) \min \left(y^{i}, M\right) \rightarrow_{\alpha \searrow 0, M \rightarrow \infty} y^{i}$ in $L_{1}^{m}$-norm, $U^{i}\left((1-\alpha) \min \left(y^{i}, M\right)\right) \rightarrow_{\alpha \searrow 0, M \rightarrow \infty} U^{i}\left(y^{i}\right)$ by Lemma 2, and thus w.1.o.g. $y^{i}$ is bounded and satisfies ${ }^{22}$

$$
\int_{\Omega} \pi \cdot y^{i} d \mu<\int_{\Omega} \pi \cdot e^{i} d \mu
$$

(otherwise it can be replaced by some $(1-\alpha) \min \left(y^{i}, M\right)$ ).

Now define $y_{k}^{i} \equiv E\left(y^{i} \mid \digamma_{k}^{i}\right)$; it is $\digamma_{k}^{i}$-measurable. Since $y_{k}^{i} \rightarrow_{k \rightarrow \infty} y^{i}$ in measure (by Theorem 4 of Boylan, 1971), $y_{k}^{i} \rightarrow \underset{k \rightarrow \infty}{L_{1}^{m}} y^{i}$ because of the uniform boundedness of $y_{k}^{i}$ and $y^{i}$, and it can be established similarly to (14) that

$$
\int_{\Omega} \pi_{k} \cdot y_{k}^{i} d \mu \rightarrow_{k \rightarrow \infty} \int_{\Omega} \pi \cdot y^{i} d \mu .
$$

Together with (15) and (16) this implies that $y_{k}^{i} \in B^{i}\left(\pi_{k}\right)$ for all sufficiently large $k$. But $U^{i}\left(y^{i}\right)>U^{i}\left(x^{i}\right)$, and $x_{k}^{i} \rightarrow{ }_{k \rightarrow \infty}^{L_{1}^{m}} x^{i}, y_{k}^{i} \rightarrow_{k \rightarrow \infty}^{L_{1}^{m}} y^{i}$. By Lemma 2, for all sufficiently large $k$

$$
U_{k}^{i}\left(y_{k}^{i}\right)>U_{k}^{i}\left(x_{k}^{i}\right),
$$

${ }^{22}$ We use the fact that $\int_{\Omega} \pi \cdot e^{i}>0$, which is due to strict positivity of $e^{i}$, and positivity of the non-vanishing $\pi$. 
where $U_{k}^{i}$ is defined by (2) for the utility function $u_{k}$. This cannot be consistent with $y_{k}^{i} \in B^{i}\left(\pi_{k}\right)$ since $\left(\mathbf{x}_{k}, \pi_{k}\right) \in W E E\left(\mathcal{E}_{k}\right)$. We reached a contradiction, which leads to the conclusion that $(\mathbf{x}, \pi) \in W E E(\mathcal{E})$.

Proof of Theorem 2. As in the proof of Theorem $1, \mathbf{x}=\left(x^{1}, \ldots, x^{n}\right)$ is a private allocation in $\mathcal{E}$. Assume, however, that $\mathrm{x}$ is not in the private core of $\mathcal{E}$. Then there exist a non-empty coalition $S$ and a private $S$-allocation $\mathbf{y}=\left(y^{i}\right)_{i \in S}$ such that

$$
U^{i}\left(y^{i}\right)>U^{i}\left(x^{i}\right)
$$

for every $i \in S$.

We will now modify y to obtain an $S$-allocation in $\mathcal{E}_{k}$ for a certain large $k$. For every $\varepsilon>0$ denote

$$
A_{k, \varepsilon}^{i}=\left\{\omega \in \Omega \mid\left\|e_{k}^{i}(\omega)-e^{i}(\omega)\right\|<\varepsilon\right\},
$$

and let $k(\varepsilon)$ be some positive integer such that

$$
\mu\left(A_{k, \varepsilon}^{i}\right)>1-\varepsilon \text { for every } i \in S
$$

holds for all $k \geq k(\varepsilon)$. Such $k(\varepsilon)$ exists for every $\varepsilon>0$, since $e_{k}^{i} \rightarrow_{k \rightarrow \infty}^{L_{1}^{m}} e^{i}$ and hence $e_{k}^{i} \rightarrow_{k \rightarrow \infty} e^{i}$ in measure. For this reason, we can also choose $\{k(\varepsilon)\}_{\varepsilon>0}$ in a way that $k(\varepsilon)$ is non-increasing in $\varepsilon$ and $\lim _{\varepsilon \searrow_{0}} k(\varepsilon)=\infty^{23}$. Clearly, for almost every $\omega \in \Omega$ and every commodity $j$

$$
\sum_{i \in S} y^{i}(\omega) \cdot \chi_{A_{k(\varepsilon), \varepsilon}^{i}}(\omega) \leq \sum_{i \in S} e_{k(\varepsilon)}^{i}(\omega)+n \varepsilon \overline{1},
$$

where $\overline{1}=(1, \ldots, 1) \in R^{m}$ (this inequality follows from the fact that $\mathbf{y}$ satisfies the feasibility constraint in $\mathcal{E}$ and the definition of $\left.A_{k(\varepsilon), \varepsilon}^{i}\right)$. Therefore, $\mathbf{y}_{k(\varepsilon)}=$ $\left(y_{k(\varepsilon)}^{i}\right)_{i \in S}$, defined by

$$
y_{k(\varepsilon)}^{i}(\omega)_{j} \equiv \max \left(y^{i}(\omega)_{j} \cdot \chi_{A_{k(\varepsilon), \varepsilon}^{i}}(\omega)-n \varepsilon, 0\right)
$$

for every $\omega \in \Omega$ and every commodity $j$, satisfies the feasibility constraint in $\mathcal{E}_{k(\varepsilon)}$ :

$$
\sum_{i \in S} y_{k(\varepsilon)}^{i}(\omega) \leq \sum_{i \in S} e_{k(\varepsilon)}^{i}(\omega) \text { for almost every } \omega \in \Omega
$$

Note that each $A_{k, \varepsilon}^{i}$ is $\digamma_{k}^{i}$-measurable (since both $e_{k}^{i}$ and $e^{i}$ are $\digamma_{k}^{i}$-measurable due to the inclusion $\digamma_{k}^{i} \supseteq \digamma^{i}$ ), and so $y_{k(\varepsilon)}^{i}$ is $\digamma_{k(\varepsilon)}^{i}$-measurable. From this fact and (19) it follows that the sequence $\left\{\mathbf{y}_{k\left(\frac{1}{r}\right)}\right\}_{r=1}^{\infty}$ is a sequence of private $S$-allocations in economies $\left\{\mathcal{E}_{k\left(\frac{1}{r}\right)}\right\}_{r=1}^{\infty}$, and (18) implies that for each $i \in S\left\{y_{k\left(\frac{1}{r}\right)}^{i}\right\}_{r=1}^{\infty}$ converges to $y^{i}$ in measure.

Since for each $i \in S$ and $r y_{k\left(\frac{1}{r}\right)}^{i} \leq y^{i}$, the sequence $\left\{y_{k\left(\frac{1}{r}\right)}^{i}\right\}_{r=1}^{\infty}$ is bounded from above by an integrable function. Therefore, its convergence in measure to

23 Or, at least, that $k\left(\frac{1}{r}\right)$ is non-decreasing when $r$ is a positive integer, and $\lim _{r \rightarrow \infty} k\left(\frac{1}{r}\right)=\infty$. 
$y^{i}$ implies convergence in the $L_{1}^{m}$-norm as well. However, according to this and Lemma 2, for every $i \in S$

$$
\lim _{r \rightarrow \infty} U_{k\left(\frac{1}{r}\right)}^{i}\left(y_{k\left(\frac{1}{r}\right)}^{i}\right)=U^{i}\left(y^{i}\right) .
$$

Also, since $x_{k}^{i} \rightarrow \rightarrow_{k \rightarrow \infty}^{L_{1}^{m}} x^{i}$, for every $i \in S$

$$
\lim _{r \rightarrow \infty} U_{k\left(\frac{1}{r}\right)}^{i}\left(x_{k\left(\frac{1}{r}\right)}^{i}\right)=U^{i}\left(x^{i}\right) .
$$

Due to assumption (17), these two equalities yield existence of $r$ for which

$$
U_{k\left(\frac{1}{r}\right)}^{i}\left(y_{k\left(\frac{1}{r}\right)}^{i}\right)>U_{k\left(\frac{1}{r}\right)}^{i}\left(x_{k\left(\frac{1}{r}\right)}^{i}\right)
$$

holds for every $i \in S$. This contradicts the assumption that $\mathbf{x}_{k\left(\frac{1}{r}\right)}$ is a private core allocation in $\mathcal{E}_{k\left(\frac{1}{r}\right)}$.

Proof of Theorem 3. As in the proof of Theorem 2, if the limit allocation $\mathbf{x}=$ $\left(x^{1}, \ldots, x^{n}\right)$ is not in the private core of $\mathcal{E}$, there exist a non-empty coalition $S$ and a private $S$-allocation $\mathbf{y}=\left(y^{i}\right)_{i \in S}$ such that

$$
U^{i}\left(y^{i}\right)>U^{i}\left(x^{i}\right)
$$

for every $i \in S$. We can assume w.l.o.g. that each $y^{i}$ is bounded. (Otherwise it can be replaced by $\min \left(y^{i}, M\right)$, which converges to $y^{i}$ in $L_{1}^{m}$-norm as $M \rightarrow \infty$. Indeed, according to Lemma $2, U^{i}\left(\min \left(y^{i}, M\right)\right) \rightarrow_{M \rightarrow \infty} U^{i}\left(y^{i}\right)$, and thus this replacement will leave (20) intact for sufficiently large $M$. Clearly, the replacement also leads to a private allocation.) We will show next that it can also be assumed w.l.o.g. that each $y^{i}$ is continuous.

By Lusin theorem, for every $\varepsilon>0$ and $i \in S$ there exists a continuous function $y_{\varepsilon}^{i}$ on $\Omega$, such that $\mu\left(A_{\varepsilon}^{i}\right)<\varepsilon$ where $A_{\varepsilon}^{i}=\left\{\omega \mid y^{i}(\omega) \neq y_{\varepsilon}^{i}(\omega)\right\}$. Also denote

$$
B_{\varepsilon}=\left\{\omega \mid \text { there exists } j \text { with } \sum_{i \in S} y_{\varepsilon}^{i}(\omega)_{j} \geq \sum_{i \in S} e^{i}(\omega)_{j}+\varepsilon\right\}
$$

and

$$
B=\left\{\omega \mid \sum_{i \in S} y_{\varepsilon}^{i}(\omega) \leq \sum_{i \in S} e^{i}(\omega)\right\} .
$$

Since these subsets of a compact metric space $\Omega$ are disjoint and closed (here we use continuity of both $e^{i}$ and $y_{\varepsilon}^{i}$ for every $i \in S$ ), they can be separated, i.e., there is a continuous function $c: \Omega \rightarrow[0,1]$ such that $c \equiv 1$ on $B$ and $c \equiv 0$ on $B_{\varepsilon}$. Thus, $\widetilde{\mathbf{y}}_{\varepsilon}=\left(\widetilde{y}_{\varepsilon}^{i}\right)_{i \in S}$ given by

$$
\widetilde{y}_{\varepsilon}^{i}(\omega)_{j} \equiv \max \left(c(\omega) \cdot y_{\varepsilon}^{i}(\omega)_{j}-\varepsilon, 0\right)
$$

for every $i \in S$, commodity $j$, and $\omega \in \Omega$, satisfies the feasibility constraint in $\mathcal{E}$ :

$$
\sum_{i \in S} \widetilde{y}_{\varepsilon}^{i}(\omega) \leq \sum_{i \in S} e^{i}(\omega) \text { for every } \omega \in \Omega .
$$


Since each $\widetilde{y}_{\varepsilon}^{i}$ is $\digamma^{i}$-measurable as a continuous function on $\Omega$ (recall that $\digamma^{i}=\digamma$ is the $\sigma$-field of all Borel sets in $\Omega$ ), $\widetilde{\mathbf{y}}_{\varepsilon}$ is in fact a private $S$-allocation that consists of continuous functions. Moreover, for each $i \in S$ and a commodity $j$

$$
y_{\varepsilon}^{i}(\omega)_{j}-\varepsilon \leq \widetilde{y}_{\varepsilon}^{i}(\omega)_{j} \leq y_{\varepsilon}^{i}(\omega)_{j}
$$

for almost every $\omega \in \bigcap_{i \in S}\left(A_{\varepsilon}^{i}\right)^{c}$, since $\mathbf{y}$ satisfies the feasibility constraint $\sum_{i \in S} y^{i}(\omega) \leq \sum_{i \in S} e^{i}(\omega)$ for almost every $\omega \in \Omega$ and hence $\mu\left(\bigcap_{i \in S}\left(A_{\varepsilon}^{i}\right)^{c} \backslash B\right)=0$. Clearly $\mu\left(\bigcap_{i \in S}\left(A_{\varepsilon}^{i}\right)^{c}\right)>1-n \varepsilon$, and together with (21) this implies that functions in $\widetilde{\mathbf{y}}_{\varepsilon}$ converge in measure to those in $\mathbf{y}$ as $\varepsilon \searrow 0$. From the (obvious) uniform boundedness ${ }^{24}$ of $\widetilde{\mathbf{y}}_{\varepsilon}$ and $\mathbf{y}$ it follows that the convergence is in the $L_{1}^{m}$-norm as well. By Lemma 2 and (20),

$$
U^{i}\left(\widetilde{y}_{\varepsilon_{0}}^{i}\right)>U^{i}\left(x^{i}\right)
$$

for every $i \in S$ and some sufficiently small $\varepsilon_{0}$. Thus, by replacing $\mathbf{y}$ in (20) by $\widetilde{\mathbf{y}}_{\varepsilon_{0}}$ if necessary, we can w.l.o.g. assume that (20) holds for $y$ that consists of continuous functions.

For every $k=1,2, \ldots$ and $i \in S$ define $z_{k}^{i}=E\left(y^{i} \mid \digamma_{k}^{i}\right) ; z_{k}^{i}$ is clearly $\digamma_{k}^{i}$ measurable and is (or can be chosen to be) bounded by the same constant as $y^{i}$. We will show that a subsequence of $\left\{z_{k}^{i}\right\}_{k=1}^{\infty}$ converges to $y^{i}$ uniformly (almost everywhere). For every commodity $j$ and two positive integers $K, l$ with $0 \leq l \leq K$, consider a pair of closed ${ }^{25}$ subsets of $\Omega$ :

$$
C_{K}^{l}(j)=\left\{\omega \mid \frac{l-1}{K} \max _{\omega \in \Omega} y_{j}^{i} \leq y^{i}(\omega)_{j} \leq \frac{l}{K} \max _{\omega \in \Omega} y_{j}^{i}\right\}
$$

and

$$
\widetilde{C}_{K}^{l}(j)=\left\{\omega \mid \frac{l-2}{K} \max _{\omega \in \Omega} y_{j}^{i} \geq y^{i}(\omega)_{j} \text { or } \frac{l+1}{K} \max _{\omega \in \Omega} y_{j}^{i} \leq y^{i}(\omega)_{j}\right\} .
$$

Since $\digamma_{k}^{i}$ can separate disjoint closed sets for all sufficiently large $k$, for every $K$ there exists $k=k(K)$ independent of $i$ and $j$ such that $\digamma_{k(K)}^{i}$ separates $C_{K}^{l}(j)$ from $\widetilde{C}_{K}^{l}(j)$ for every $j$ and $1 \leq l \leq K$; it can also be assumed that $\lim _{K \rightarrow \infty} k(K)=\infty$. Thus for every $j$ and $1 \leq l \leq K$ there is a set $D_{K}^{l}(j) \in \digamma_{k(K)}^{i}$ such that

$$
\left\{\omega \mid \frac{l-1}{K} \max _{\omega \in \Omega} y_{j}^{i} \leq y^{i}(\omega)_{j} \leq \frac{l}{K} \max _{\omega \in \Omega} y_{j}^{i}\right\} \subset D_{K}^{l}(j)
$$

(and hence $D_{K}^{1}(j), D_{K}^{2}(j), \ldots, D_{K}^{K}(j) \operatorname{cover} \Omega$ ), and

$$
D_{K}^{l}(j) \subset\left\{\omega \mid \frac{l-2}{K} \max _{\omega \in \Omega} y_{j}^{i}<y^{i}(\omega)_{j}<\frac{l+1}{K} \max _{\omega \in \Omega} y_{j}^{i}\right\} .
$$

Consequently, for every $j$ and $1 \leq l \leq K$, and almost every $\omega \in D_{K}^{l}(j)$,

$$
z_{k(K)}^{i}(\omega)_{j}-y^{i}(\omega)_{j}=E\left(y_{j}^{i} \mid \digamma_{k(K)}^{i}\right)(\omega)-y^{i}(\omega)_{j}
$$

${ }^{24}$ Here we use the (w.l.o.g.) assumption that the commodity bundles in $\mathbf{y}$ are bounded functions.

25 Closedness is due to the continuity of $y^{i}$. 
(here we use the fact that $D_{K}^{l}(j) \in \digamma_{k(K)}^{i}$ )

$$
\begin{aligned}
& =E\left(y_{j}^{i} \cdot \chi_{D_{K}^{l}(j)} \mid \digamma_{k(K)}^{i}\right)(\omega)-y^{i}(\omega)_{j} \\
& \leq E\left(\frac{l+1}{K} \max _{\omega \in \Omega} y_{j}^{i} \cdot \chi_{D_{K}^{l}(j)} \mid \digamma_{k(K)}^{i}\right)(\omega)-\frac{l-2}{K} \max _{\omega \in \Omega} y_{j}^{i} \\
& =\frac{l+1}{K} \max _{\omega \in \Omega} y_{j}^{i}-\frac{l-2}{K} \max _{\omega \in \Omega} y_{j}^{i}=\frac{3}{K} \max _{\omega \in \Omega} y_{j}^{i} .
\end{aligned}
$$

Similarly,

$$
z_{k(K)}^{i}(\omega)_{j}-y^{i}(\omega)_{j} \geq-\frac{3}{K} \max _{\omega \in \Omega} y_{j}^{i}
$$

for almost every $\omega \in D_{K}^{l}(j)$. We conclude that

$$
\left|z_{k(K)}^{i}(\omega)_{j}-y^{i}(\omega)_{j}\right| \leq \frac{3}{K} \max _{\omega \in \Omega} y_{j}^{i} \equiv \delta_{K}(j) \text { for almost every } \omega \in \Omega .
$$

(This means that $\left\{z_{k(K)}^{i}\right\}_{K=1}^{\infty}$ converges to $y^{i}$ uniformly (almost everywhere).) Thus

$$
\sum_{i \in S} z_{k(K)}^{i}(\omega)_{j} \leq \sum_{i \in S} y^{i}(\omega)_{j}+n \delta_{K}(j) \leq \sum_{i \in S} e^{i}(\omega)_{j}+n \delta_{K}(j)
$$

for almost every $\omega \in \Omega$.

Now denote

$$
y_{k(K)}^{i}(\omega)_{j} \equiv \max \left(z_{k(K)}^{i}(\omega)_{j}-n \delta_{K}(j), 0\right)
$$

for every $i \in S$, commodity $j$, and $\omega \in \Omega$. It is clear that $\left\{y_{k(K)}^{i}\right\}_{K=1}^{\infty}$ converges to $y^{i}$ uniformly, and that (from (22)) the feasibility constraint

$$
\sum_{i \in S} y_{k(K)}^{i}(\omega)_{j} \leq \sum_{i \in S} e^{i}(\omega)_{j}
$$

is satisfied for every $j$ and almost every $\omega \in \Omega$. Each $y_{k(K)}^{i}$ is also $\digamma_{k(K)^{-}}^{i}$ measurable, since so is $z_{k(K)}^{i}$. We conclude that $\left\{\mathbf{y}_{k(K)}\right\}_{K=1}^{\infty}$, where $\mathbf{y}_{k(K)}=$ $\left(y_{k(K)}^{i}\right)_{i \in S}$, is a sequence of private $S$-allocations in economies $\left\{\mathcal{E}_{k(K)}\right\}_{K=1}^{\infty}$. Uniform convergence of functions implies convergence in $L_{1}^{m}$-norm, and hence, according to Lemma 2, for every $i \in S$

$$
\lim _{K \rightarrow \infty} U_{k(K)}^{i}\left(y_{k(K)}^{i}\right)=U^{i}\left(y^{i}\right) \text { and } \lim _{K \rightarrow \infty} U_{k(K)}^{i}\left(x_{k(K)}^{i}\right)=U^{i}\left(x^{i}\right) .
$$

Due to (20), there exists $K$ such that

$$
U_{k(K)}^{i}\left(y_{k(K)}^{i}\right)>U_{k(K)}^{i}\left(x_{k(K)}^{i}\right)
$$

for every $i \in S$. This contradicts the assumption that $\mathbf{x}_{k(K)}$ is a private core allocation in $\mathcal{E}_{k(K)}$. 


\section{References}

Allen, B.: Neighboring information and distribution of agent characteristics under uncertainty. Journal of Mathematical Economics 12, 63-101 (1983)

Allen, B., Yannelis, N.C.: Differential information economies: introduction. Economic Theory 18, 263 274 (2001)

Balder, E.J., Yannelis, N.C.: Continuity properties of the core in games with differential information. Memo (2002)

Boylan, E.: Equiconvergence of martingales. Annals of Mathematical Statistics 42, 552-559 (1971)

Cotter, K.D.: Similarity of information and behavior with pointwise convergence topology. Journal of Mathematical Economics 15, 25-38 (1986)

Cotter, K.D.: Convergence of information, random variable and noise. Journal of Mathematical Economics 16, 39-51 (1987)

Einy E., Moreno, D., Shitovitz, B.: Competitive and core allocations of large economies with differential information. Economic Theory 18, 321-332 (2001)

Forges, F., Minelli, E., Vohra, R.: Incentive and the core of an exchange economy: a survey. Journal of Mathematical Economics 38, 1-41 (2002)

Glycopantis D., Muir, A., Yannelis, N.C.: An extensive form interpretation of the private core. Economic Theory 18, 293-319 (2001)

Hildenbrand, K.: Continuity of the equilibrium set correspondence. Journal of Economic Theory 5, 152-161 (1972)

Hildenbrand, W., Mertens, J.F.: Upper hemi-continuity of the equilibrium set correspondence for pure exchange economies. Econometrica 40, 99-108 (1972)

Kannai, Y.: Continuity properties of the core of a market. Econometrica 38, 791-815 (1970)

Krasa, S., Shafer, W.: Core concepts in economies where information is almost complete. Economic Theory 18, 451-471 (2001)

Neveu, J.: Mathematical foundations of the calculus of probability. San Francisco: Holden Day 1965

Radner, R.: Competitive equilibrium under uncertainty. Econometrica 36, 31-58 (1968)

Radner, R.: Equilibrium under uncertainty. In: Arrow, K.J. Intrilligator, M.D. (eds.) Handbook of mathematical economics, vol. II. Amsterdam: North Holland 1982

Stinchcombe, M.: Bayesian information topologies. Journal of Mathematical Economics 19, 233-253 (1990)

Van Zandt, T.: Information, measurability, and continuous behavior. Journal of Mathematical Economics 38, 293-309 (2002)

Vohra, R.: Incomplete information, incentive compatibility and the core. Journal of Economic Theory 86, 123-147 (1999)

Wilson, R.: Information, efficiency, and the core of an economy. Econometrica 46, 807-816 (1978)

Yannelis, N.C.: The Core of an Economy with Differential Information, Economic Theory 1, 183-198 (1991) 